\title{
A Expectativa Profissional do Futuro Médico: Análise do Quadriênio 2014-2017
}

\section{Professional Expectations of Future Doctors: Analysis of the Quadriennium 2014-2017}

\author{
Luciano Moura de AssunçãoI \\ Ana Beatriz Cardoso Pereira ${ }^{I}$ \\ Lana Carla Fernandes de Albuquerquel \\ Luana Bastos de Mont' Alverne Ferreira ${ }^{I}$ \\ Cezar Augusto Muniz Caldas ${ }^{I}$
}

\section{PALAVRAS-CHAVE}

- Educação Médica.

- Prática Profissional.

- Escolha da Profissão.

\section{RESUMO}

O objetivo deste trabalho é descrever as expectativas dos estudantes de Medicina quanto à carreira profissional. Foi realizado um estudo transversal, descritivo e analítico, no período de janeiro de 2014 a dezembro de 2017, com 116 estudantes concluintes em 2014, 116 de 2015, 91 de 2016 e 110 de 2017, totalizando uma amostra de 431 sujeitos. Foi aplicado um questionário para obtenção de informações sobre idade, gênero, ano de formatura, desejos de atuação profissional para os dez anos seguintes, a especialidade que deseja seguir, o nível de atenção no qual deseja trabalhar, a renda a ser alcançada, o número de empregos que acha necessário para alcançá-la e o número de empregos que pretende ter. $O$ estudo demonstrou predomínio de homens (58,7\%), e a média de idade foi de 26,4 $\pm 3,91$ anos. Entre as especialidades pretendidas pelos egressos, Clínica Médica foi a mais almejada em todos os anos estudados, exceto no ano de 2016, em que predominou Pediatria. Sobre os desejos profissionais para dez anos após a formação, a docência foi almejada por cerca da metade deles, variando entre 46\% e 59\%, enquanto a quase totalidade da amostra demonstrou o desejo de trabalhar com assistência (atendimento direto ao paciente). Não houve predominio entre as atividades ambulatorial/consultório e hospitalar, assim como entre o setor público e o privado. Em relação ao desejo de atuar nos diferentes níveis de atenção à saúde, os formandos de 2014 e 2016 expressaram desejo de atuar predominantemente no nível terciário $(40,5 \pm 29,3 \%$ e 41,1 $\pm 29,7 \%$, respectivamente) em relação aos demais níveis de atenção. Sobre a pretensão de renda salarial, predominou a faixa acima de dez salários mínimos, sendo que a maioria expressou achar necessários pelo menos três empregos para alcançá-la, embora o desejo da maior parte dos estudados tenha sido manter apenas dois empregos. Conclui-se que as chamadas áreas básicas, como Clínica Médica, Cirurgia Geral e Pediatria, ainda são bastante almejadas pelos formandos. Contudo, mais do que o desejo de segui-las, provavelmente isto representa a necessidade de pré-requisito para acesso a outras especialidades. No geral, os egressos pretendem trabalhar com assistência, têm uma expectativa salarial alta, admitem a necessidade de diversos vínculos empregatícios para atingir esta meta e têm pouco interesse em trabalhar no nível primário de atenção à saúde. Tal realidade poderia ser discutida no âmbito da academia, a fim de oferecer informações realistas aos estudantes sobre o mercado de trabalho e a carreira médica, minimizando frustrações futuras durante o exercício profissional. 


\section{KEY-WORDS}

- Medical Education.

- Professional Practice.

- Career Choice.

Recebido em: 12/2/19

Aceito em: 27/2/19
ABSTRACT

The objective of this work is to describe the expectations of medical students regarding their professional career. In order to do this, a cross-sectional descriptive and analytical study was conducted, from January 2014 to December 2017, with 116 senior students of the class of 2014, 116 of 2015, 91 of 2016 and 110 of 2017, with a total of 431 individuals. A survey was applied to obtain information about their age, gender, graduation year, professional expectations for the 10 years to follow, intended specialty, level of care at which they want to work, intended income, the number of jobs they think they will need to work in to reach that level of income and the number of jobs they expect to have. The study showed that there are more male students (58\%) than female, and the average age is $26.4 \pm$ 3.91 years old. General Medicine was the most sought specialty by the graduate students, except for those of the year 2016, when the predominant specialty was Pediatrics. Concerning the professional goals for the 10 years after graduation, teaching was an objective of approximately half of them, ranging between $46 \%$ and $59 \%$, while almost all of them demonstrated a wish to perform assistance work (direct patient care). No predominance was found when comparing the expectation of working in a hospital or in private clinics, nor between the private and public sector. Concerning the wish to work at different health care levels, the senior students from the classes of 2014 and 2016 expressed their wish to work predominantly at care level $3(40.5 \pm 29.3 \%$ and $41.1 \pm 29.7 \%$, respectively). Concerning the intended income, the most popular range was above 10 minimum monthly salaries. Most students stated they believe that at least three jobs would be necessary to reach this salary level, but they also expressed the wish of having only two jobs. We conclude that the so-called basic areas, such as General Medicine, General Surgery and Pediatrics are the most coveted by the senior students. However, this is probably due not only to a desire to follow these specialties, but also because they represent a prerequisite to access other specialties. In general, the graduates intend to work with direct care and have high salary expectations, which is only achieved by having multiple jobs. They have little interest in working at care level one. This reality could be discussed in academic circles, in order to offer realistic information to students about the job market and medical career, minimizing future frustrations during their professional practice.

\section{INTRODUÇÃO}

O passar dos anos traz mudanças sociais, econômicas e culturais inevitáveis, e, nesse contexto, estão inseridos os cursos de Medicina, responsáveis por fornecer médicos capacitados para atuar nessa realidade. No Brasil, diversas escolas de Medicina foram abertas nos últimos anos ${ }^{1,2}$, porém pouco se conhece sobre as áreas de atuação de seus egressos, empregabilidade e educação continuada para atendimento às crescentes demandas populacionais no País ${ }^{3}$. Frente à realidade do mercado de trabalho, encontram-se as expectativas do estudante concluinte do curso de Medicina. Uma importante questão a ser esclarecida é se tais expectativas são coerentes com o perfil desejado para o egresso da escola médica, a fim de elucidar se a escola está conseguindo formar o profissional com o perfil desejado, compatível com as Diretrizes Curriculares Nacionais de $2014^{4}$.
A literatura indica uma crise global de escassez grave e má distribuição de profissionais de saúde, especialmente influenciadas por fatores como alterações demográficas e mudanças epidemiológicas ${ }^{5}$. Será que os egressos dos cursos de Medicina do Brasil estão sensíveis a este cenário e estão dispostos a enfrentar a nova realidade que se impõe? Quanto ao aspecto da má distribuição de profissionais de saúde, sendo esta uma condição global e de maneira alguma exclusiva do Brasil, as populações rurais remotas e pobres não são, muitas vezes, capazes de atrair ou reter profissionais de saúde. Contudo, há indícios de que um maior número de profissionais pode ser mantido em áreas rurais por meio de políticas no setor da educação (localização de escolas médicas e de enfermagem, política de admissões - sistema de cotas ou ações afirmativas -, bolsas de estudo e exigência de um período de serviço em uma área rural após concluir a formação) e no setor da saúde 
(remuneração adequada, rede de ensino para crianças e desenvolvimento de planos de carreira) $)^{5,6}$.

Nota-se aí o papel fundamental tanto da escola médica como dos gestores em saúde para garantir a formação de profissionais de acordo com as necessidades da população.

Em 2010, a Comissão sobre educação dos profissionais de saúde para o século XXI já alertava sobre a necessidade de reformular a formação do profissional de saúde, devendo-se educar os profissionais para mobilizarem conhecimentos e se engajarem em raciocínio crítico e conduta ética, para participar de maneira competente e responsável dos sistemas de saúde centrados na população e no paciente ${ }^{2}$.

Além disso, a transição universidade-trabalho é problemática, multifatorial e dinâmica, pois se trata de um momento fecundo de ideias e aflições para o estabelecimento de novas metas profissionais, o que implica planejar o futuro, reavaliar as escolhas e antecipar o que está por vir em termos profissionais e pessoais ${ }^{7}$. O último ano do curso de Medicina é repleto de ansiedades e incertezas, sendo importante para o aluno e para a instituição conhecer as dificuldades de inserção no mercado de trabalho, os problemas ligados à sua formação, preparo (ou despreparo) em diferentes tópicos, por exemplo ${ }^{8}$.

Assim, nos dias atuais, a averiguação das características dos ex-alunos tem sido recomendada nas políticas de recursos humanos das instituições de saúde ${ }^{9}$. Com base nisto, o presente estudo tem como principal objetivo descrever as expectativas dos estudantes de Medicina quanto à carreira profissional.

\section{METODOLOGIA}

Foi realizado um estudo transversal, descritivo e analítico, no período de janeiro de 2014 a dezembro de 2017, entre concluintes do curso de Medicina da Universidade Federal do Pará (UFPA).

Os estudantes foram abordados antes do início da última prova do último semestre do curso, quando foram orientados sobre os objetivos, riscos e benefícios da pesquisa. Daqueles que concordaram em participar da pesquisa, foi solicitado o consentimento por meio da assinatura do Termo de Consentimento Livre e Esclarecido (TCLE) e do preenchimento do questionário elaborado pelos autores, para obtenção de informações sobre idade; sexo; ano de formatura; desejos de atuação profissional para os dez anos seguintes; especialidade que deseja seguir; nível de atenção em que deseja trabalhar; renda a ser alcançada; e número de empregos que pretende ter. Os pesquisadores ficaram à disposição durante o preenchimento dos questionários, a fim de dirimir eventuais dúvidas dos estudantes.

A UFPA forma cerca de 150 estudantes por ano, sendo incluídos neste estudo os questionários preenchidos por 116 estudantes concluintes em 2014, 116 estudantes de 2015, 91 de 2016 e 110 de 2017. Foram incluídos estudantes concluintes do curso de Medicina naquele ano e excluídos os questionários com preenchimento incorreto.

O estudo foi aprovado pelo Comitê de Ética em Pesquisa do Instituto de Ciências da Saúde (ICS), sob o no 319.222 em 28/06/2013 (CAAE 16134813.0.0000.0018), renovado em 29/04/2015 (Parecer no 1.043.440) e em 13/07/2017 (Parecer $\left.\mathrm{n}^{\circ} 2.170 .864\right)$. Os dados recolhidos foram organizados e analisados por meio de planilhas do Microsoft Excel 2007®. As variáveis categóricas foram apresentadas em valores absolutos e percentuais, sendo utilizado o teste do qui-quadrado para avaliar a significância dos resultados. As variáveis contínuas foram expressas como média \pm desvio-padrão, sendo utilizado o teste de Anova para verificar diferenças entre os grupos. $\mathrm{O}$ valor de $p<0,05$ foi considerado estatisticamente significativo.

\section{RESULTADOS}

A amostra composta por egressos do curso de Medicina da UFPA entre os anos de 2014 e 2017 totalizou 431 sujeitos. Destes, 116 egressos eram de 2014 (26,9\%), 114 de 2015 (26,5\%), 91 de 2016 (21,1\%) e 110 de 2017 (25,5\%), com média de idade de 26,4 anos e predomínio estatisticamente significante do gênero masculino (Tabela 1).

\section{TABELA 1}

Descrição conforme gênero, idade e ano de formatura dos egressos de Medicina dos anos de 2014 a 2017 da UFPA

$\begin{array}{lccc}\text { Variáveis } & & \text { Frequência } & \% \\ \text { Sexo } & & & \\ & \text { Feminino } & 178 & 41,3 \\ & \text { Masculino* } & 253 & 58,7 \\ & \text { Total } & 431 & 100,0\end{array}$

Ano de formatura

$\begin{array}{ccc}2014 & 116 & 26,9 \\ 2015 & 114 & 26,5 \\ 2016 & 91 & 21,1 \\ 2017 & 110 & 25,5 \\ \text { Total } & 431 & 100,0\end{array}$

* $p=0,0004$ Teste Qui-Quadrado Aderência. Fonte: Protocolo de pesquisa.

A Tabela 2 destaca as cinco principais especialidades pretendidas pelos estudantes. A especialidade de Clínica Médica foi a mais almejada pelos egressos no período estudado, exceto no ano de 2016, no qual predominou a especialidade de Pediatria. 


\section{TABELA 2}

Principais especialidades médicas pretendidas pelos

egressos de Medicina dos anos de 2014 a 2017 da UFPA

Especialidade Frequência $\%$

$2014(\mathrm{n}=116)$

$\begin{array}{ccc}\text { Clínica Médica } & 20 & 17,2 \\ \text { Cirurgia Geral } & 15 & 12,9 \\ \text { Pediatria } & 14 & 12,1 \\ \text { Dermatologia } & 13 & 11,2 \\ \text { ia / Ginecologia e Obstetrícia } & 12 & 10,3\end{array}$

Anestesiologia / Ginecologia e Obstetrícia $\quad 12 \quad 10,3$

$2015(\mathrm{n}=114)$

$\begin{array}{lll}\text { Clínica Médica } & 33 & 28,9 \\ \quad \text { Pediatria } & 19 & 16,7 \\ \text { Cirurgia Geral } & 12 & 10,5 \\ \text { logia / Medicina de Família } & & \\ \text { e Comunidade } & 11 & 9,6 \\ \text { Oftalmologia } & 10 & 8,8\end{array}$

$2016(\mathrm{n}=91)$

$\begin{array}{ccc}\text { Pediatria } & 15 & 16,5 \\ \text { Clínica Médica } & 13 & 14,3 \\ \text { Neurologia } & 11 & 12,1 \\ \text { Oftalmologia } & 9 & 9,9\end{array}$

Medicina de Família e Comunidade /

Neurocirurgia / Otorrinolaringologia 8 8,8 $2017(\mathrm{n}=110)$

$\begin{array}{ccc}\text { Clínica Médica } & 19 & 17,3 \\ \text { Pediatria } & 16 & 14,5 \\ \text { Cirurgia Geral } & 15 & 13,6 \\ \text { Cirurgia Vascular /Oftalmologia } & 14 & 12,7 \\ \text { Dermatologia } & 9 & 8,2\end{array}$

Total $(\mathrm{n}=431)$

$\begin{array}{ccc}\text { Clínica Médica } & 85 & 19,7 \\ \text { Pediatria } & 64 & 14,8 \\ \text { Cirurgia Geral } & 49 & 11,4 \\ \text { Oftalmologia } & 43 & 10,0 \\ \text { Dermatologia } & 36 & 8,4\end{array}$

Fonte: Protocolo de pesquisa.

A docência foi desejada por aproximadamente metade da casuística em todos os anos analisados, variando entre $46 \%$ e $59 \%$, contrastando com o trabalho com assistência, que estava entre os desejos da quase totalidade dos egressos. Entre os participantes do estudo que desejavam trabalhar na assistência, não houve predomínio entre as atividades ambulatorial/ consultório ou hospitalar, havendo maior diferença percentual no ano de 2015, com predomínio da atividade ambulatorial/ consultório, com $69,2 \%$, porém não houve diferença estatística significativa. Da mesma forma, não houve predomínio do setor público sobre o privado, ou vice-versa (Tabela 3).

\begin{tabular}{|c|c|c|c|c|}
\hline $\begin{array}{r}\text { Preferências } \\
\text { pretendidas } \\
\text { entrevistados d }\end{array}$ & $\begin{array}{l}\text { TABELA } \\
\text { de modalid } \\
\text { pelos egres } \\
\text { os anos de } 2\end{array}$ & $\begin{array}{l}\text { ade d } \\
\text { os de } \\
14 \text { a }\end{array}$ & $\begin{array}{l}\text { trabalho } \\
\text { Medicina } \\
017 \text { da UFP }\end{array}$ & \\
\hline $\begin{array}{l}\text { O que desejaria } \\
\text { concretizar nos }\end{array}$ & $\begin{array}{r}\text { Docênci } \\
\text { ensino suF }\end{array}$ & $\begin{array}{l}\text { do } \\
\text { erior }\end{array}$ & $\begin{array}{r}\text { Trabalhar } \\
\text { assistên }\end{array}$ & $\begin{array}{l}\text { com } \\
\text { cia }\end{array}$ \\
\hline próximos dez anos? & Frequência & $\%$ & Frequência & $\%$ \\
\hline $2014(n=116)$ & 56 & 48,3 & 116 & 100,0 \\
\hline $2015(n=114)$ & 49 & 43,0 & 107 & 93,9 \\
\hline $2016(n=91)$ & 46 & 50,5 & 85 & 93,4 \\
\hline $2017(n=110)$ & 59 & 53,6 & 103 & 93,6 \\
\hline $\begin{array}{l}\text { Dos que desejam } \\
\text { trabalhar na assistência, }\end{array}$ & Hospit & & $\begin{array}{l}\text { Ambulat } \\
\text { Consult }\end{array}$ & brio / \\
\hline em qual local? & Frequência & $\%$ & Frequência & $\%$ \\
\hline $2014(\mathrm{n}=116)$ & 57 & 49,1 & 58 & 50,0 \\
\hline $2015(n=107)$ & 41 & 38,3 & 74 & 69,2 \\
\hline $2016(n=85)$ & 49 & 57,6 & 40 & 47,1 \\
\hline $2017(n=103)$ & 39 & 37,9 & 65 & 63,1 \\
\hline Dos que desejam & Públic & & Privad & \\
\hline $\begin{array}{l}\text { assistência, em qual } \\
\text { setor deseja trabalhar? }\end{array}$ & Frequência & $\%$ & Frequência & $\%$ \\
\hline $2014(n=116)$ & 61 & 52,6 & 55 & 47,4 \\
\hline $2015(n=107)$ & 67 & 62,6 & 50 & 46,7 \\
\hline $2016(n=85)$ & 39 & 45,9 & 48 & 56,5 \\
\hline $2017(n=103)$ & 47 & 45,6 & 58 & 56,3 \\
\hline
\end{tabular}

Fonte: Protocolo de pesquisa.

A Tabela 4 apresenta dados a respeito do desejo de atuar nos diferentes níveis de atenção à saúde. Os sujeitos foram convidados a graduar de $0 \%$ a $100 \%$ o percentual de tempo que gostariam de se dedicar a cada nível de atenção. A análise demonstrou que os formandos de 2015 foram os que mais demonstraram interesse em atuar no nível secundário, sendo estatisticamente significante em relação aos demais anos. Também chama a atenção que, nos anos de 2014 e 2016, o desejo de atuar no nível terciário foi estatisticamente significante em relação aos demais níveis de atenção.

\begin{tabular}{|c|c|c|c|c|c|c|}
\hline \multicolumn{7}{|c|}{$\begin{array}{l}\text { Percentual de tempo que os egressos de } \\
\text { Medicina dos anos de } 2014 \text { a } 2017 \text { da UFPA } \\
\text { pretendem dedicar a cada nível de atenção }\end{array}$} \\
\hline \multirow{3}{*}{$\begin{array}{l}\text { Ano de } \\
\text { formatura } \\
2014\end{array}$} & \multicolumn{6}{|c|}{ Tempo de atuação em cada nível de atenção } \\
\hline & \multicolumn{2}{|c|}{ Primária (\%) } & \multicolumn{2}{|c|}{ Secundária (\%) } & \multicolumn{2}{|c|}{ Terciária (\%) } \\
\hline & 25,4 & $\pm 24,1$ & 34,1 & $\pm 24,4$ & $40,5^{*}$ & $\pm 29,3$ \\
\hline 2015 & 29,2 & $\pm 24,7$ & $39,8^{*}$ & $\pm 25,4$ & 31,0 & $\pm 29,0$ \\
\hline 2016 & 29,4 & $\pm 26,7$ & 29,5 & $\pm 21,9$ & $41,1^{*}$ & $\pm 29,7$ \\
\hline 2017 & 24,2 & $\pm 21,2$ & 38,5 & $\pm 25,7$ & 37,3 & $\pm 29,3$ \\
\hline
\end{tabular}

* $p=0,0005$ Teste Anova um critério.

Fonte: Protocolo de pesquisa. 
Sobre o que pretende alcançar em relação a emprego e renda, a Tabela 5 mostra predomínio das faixas salariais mais elevadas, a partir de 10,1 salários-mínimos (SM), sendo que a maioria expressou achar necessários pelo menos três empregos para alcançar esta renda, embora o desejo da maior parte dos estudados seja manter apenas dois empregos.

\section{DISCUSSÃO}

De acordo com dados sociodemográficos do Brasil, a maioria da população médica é de homens, com $54,4 \%$ do total de 414.831 profissionais em atividade em 2017, enquanto as mulheres representam $45,6 \%{ }^{10}$. Na presente pesquisa, a população de estudo apresentou-se com predomínio do gênero masculino entre os concluintes. Quando essa proporção é comparada com a realidade nacional e de algumas outras escolas, verifica-se que o processo de "feminilização da medicina" identificado naqueles universos está bem mais avançado que na UFPA. O gênero feminino como maioria da população de egressos já foi constatado em Curitiba (PR), com 50,5\% ${ }^{11}$, e em Vitória $(E S)^{12}, \operatorname{com} 50,2 \%{ }^{12}$. Por outro lado, no presente estudo, a média de idade observada foi de $26,4 \pm 3,9$ anos, seguindo a tendência nacional de formação de médicos jovens, conhecida como "rejuvenescimento do contingente médico", uma vez que a média de idade da demografia médica brasileira atualmente é de $45,4 \pm 13,7$ anos $^{10}$.
A Resolução do Conselho Nacional de Educação nº 3, de 20/06/2014, que institui as novas Diretrizes Curriculares Nacionais do Curso de Graduação em Medicina, ressalta que o graduado com formação geral em Medicina, sem especialidade, tornar-se-á "médico generalista"4. A residência médica é um importante meio de capacitação profissional do médico, sendo reconhecida como a melhor modalidade para a formação de especialistas, e atualmente a maior parte dos médicos especialistas obtém o título via conclusão de programa de residência ${ }^{13,14}$.

A literatura indica que diversos aspectos influenciam os estudantes de Medicina na tomada de decisão quanto à especialidade médica ${ }^{15-20}$. Um desses estudos, realizado numa instituição privada em 2014, revelou que, no primeiro ano do curso, fatores como influência dos pais e afinidade pela área foram mais relevantes, enquanto no último ano a renda esperada e o tempo livre foram os principais fatores na escolha da especialidade ${ }^{15}$. Embora estes dados possam gerar preocupação quanto à discrepância entre os interesses do egresso e as necessidades da população, o estudo também revela que as especialidades pretendidas mudam durante o curso, assim como os fatores que influenciam esta escolha, sendo que, por exemplo, a influência dos professores e o período de treinamento na área - fatores que podem ser trabalhados durante a graduação - são capazes de influenciar a escolha final da especialidade.

\begin{tabular}{|c|c|c|c|c|c|}
\hline \multicolumn{6}{|c|}{ TABELA 5} \\
\hline \multirow{2}{*}{ Ano de Formatura } & \multirow{2}{*}{$\mathbf{N}$} & \multicolumn{4}{|c|}{ Renda desejada na atividade profissional\#* } \\
\hline & & Até $5 \mathrm{SM}$ & 5,1 a $10 \mathrm{SM}$ & 10,1 a $20 \mathrm{SM}$ & $20 \mathrm{SM}$ ou mais \\
\hline 2014 & 115 & $11,3 \%$ & $42,6 \%$ & $46,1 \%$ & $0,0 \%$ \\
\hline 2015 & 112 & $1,8 \%$ & $27,7 \%$ & $40,2 \%$ & $32,1 \%$ \\
\hline 2016 & 91 & $3,3 \%$ & $14,3 \%$ & $36,3 \%$ & $44,0 \%$ \\
\hline \multirow[t]{3}{*}{2017} & 110 & $1,8 \%$ & $10,0 \%$ & $46,4 \%$ & $41,8 \%$ \\
\hline & \multicolumn{5}{|c|}{ Quantos empregos você gostaria de ter para alcançar a renda desejada?\# } \\
\hline & $\mathbf{N}$ & 1 & 2 & 3 & 4 ou mais \\
\hline 2014 & 115 & $19,1 \%$ & $48,7 \%$ & $28,7 \%$ & $3,5 \%$ \\
\hline 2015 & 112 & $11,6 \%$ & $60,7 \%$ & $24,1 \%$ & $5,4 \%$ \\
\hline 2016 & 91 & $9,9 \%$ & $59,3 \%$ & $25,3 \%$ & $4,4 \%$ \\
\hline \multirow[t]{3}{*}{2017} & 110 & $11,8 \%$ & $54,5 \%$ & $28,2 \%$ & $4,5 \%$ \\
\hline & \multirow{2}{*}{$\mathbf{N}$} & \multicolumn{4}{|c|}{ Quantos empregos acha necessários para alcançar a renda que deseja?\& } \\
\hline & & 1 & 2 & 3 & 4 ou mais \\
\hline 2014 & 115 & $7,0 \%$ & $23,5 \%$ & $41,7 \%$ & $27,8 \%$ \\
\hline 2015 & 114 & $5,3 \%$ & $31,6 \%$ & $43,0 \%$ & $20,2 \%$ \\
\hline 2016 & 87 & $8,0 \%$ & $31,0 \%$ & $43,7 \%$ & $17,2 \%$ \\
\hline 2017 & 109 & $3,7 \%$ & $33,9 \%$ & $45,0 \%$ & $17,4 \%$ \\
\hline
\end{tabular}

\# 3 questionários sem informação; \& 6 questionários sem informação. Fonte: Protocolo de pesquisa. 
Segundo a Demografia Médica Brasileira, a Pediatria é a especialidade com maior preferência entre os médicos recém-formados em geral ${ }^{10}$. Nesse estudo, Clínica Médica foi a especialidade mais almejada pelos egressos quando avaliados todos os anos. Somente no ano de 2016 a Pediatria foi a especialidade preferida pelos egressos, sendo a segunda especialidade mais desejada quando analisados todos os anos. Da Silva e colaboradores ${ }^{21}$, ao estudarem os fatores e motivações para a escolha da Pediatria como especialidade, destacaram a influência do sexo feminino como um fator relevante, assim como a influência dos formadores. No curso de Medicina da UFPA, a área de Pediatria tradicionalmente é a mais bem avaliada pelos estudantes nos levantamentos de opinião sobre o curso no final da graduação, sendo constante a escolha de professores da área como homenageados, o que possivelmente foi um importante fator que influenciou a escolha dos alunos.

No que concerne à busca por áreas gerais, observou-se um incremento da busca por Clínica Médica. Isto pode ser explicado pelo elevado número de especialidades que tem como pré-requisito a residência em Clínica Médica. Após essa formação geral, muitos egressos buscam áreas mais especializadas, que são consideradas fornecedoras de melhor ganho financeiro ou melhor qualidade de vida ${ }^{22}$.

As cinco especialidades com maior preferência entre os recém-formados brasileiros são: Pediatria (12,3\%), Clínica Médica $(11,5 \%)$, Cirurgia Geral (8,8\%), Ginecologia e Obstetrícia $(8,6 \%)$, Anestesiologia (7,1\%). Estas, quando somadas, representam $48,3 \%$ do total da preferência dos recém-formados ${ }^{10}$. Esta preferência divergiu do desejo dos egressos deste estudo, que, além das áreas básicas (Clínica Médica, Pediatria e Cirurgia Geral), teve entre as preferências Oftalmologia (10\%) e Dermatologia (8,4\%). Assemelha-se, no entanto, a um estudo local que envolveu três escolas médicas do Pará, cujas três especialidades mais almejadas pelos internos do quinto e sexto anos do curso foram: Cirurgia Geral (11,4\%), Pediatria $(7,5 \%)$, Oftalmologia $(7,1 \%)$ e Dermatologia $(6,7 \%)^{23}$.

A escolha da especialidade é uma importante decisão que define a carreira profissional de um médico. Diversos aspectos influenciam os estudantes de Medicina na tomada de decisão quanto à especialidade médica a ser seguida. Estes podem variar de acordo com componentes individuais, como, por exemplo, personalidade e experiências vivenciadas na especialidade almejada. A autonomia sobre a própria vida, um estilo de vida chamado "controlável", tornou-se um elemento decisivo nos critérios de escolha da especialidade pelos alunos ${ }^{18,24}$. Em um estudo realizado em 2014 entre estudantes de Medicina no final do curso, o fator predominante na escolha da especialidade foi a renda financeira que esta poderia proporcionar ${ }^{15}$.
Apesar dos esforços dos ministérios da Saúde e da Educação em formar um profissional capacitado para ações de promoção, prevenção, recuperação e reabilitação à saúde, na perspectiva da integralidade da assistência e promotor da saúde integral do ser humano, segundo a Demografia Médica Brasileira, apenas 1,5\% dos médicos recém-formados têm como primeira opção cursar residência médica em Medicina da Família e Comunidade. Caminhando no mesmo sentido, o presente estudo mostrou que Medicina da Família e Comunidade representava apenas $4,4 \%$ do desejo de especialização pelos egressos da UFPA no momento de sua formação.

$\mathrm{Na}$ perspectiva de conceber profissionais de saúde voltados para um sistema público que atenda às principais demandas de saúde da sociedade, a falta de profissionais com interesse pela Medicina da Família e Comunidade necessita de atenção da academia e dos gestores de saúde. Sabe-se que a educação profissional é profundamente afetada pelo ambiente disponível para treinamento clínico, sendo que o currículo acadêmico pode favorecer o viés de uma educação em instalações de nível terciário, tanto quanto favorecer uma gama de práticas em comunidade, nos domicílios ou em outros locais que incluam a população menos favorecida ${ }^{2}$. No curso de Medicina da UFPA, o eixo de atenção integral à saúde procura oferecer vivências e práticas em unidades de saúde urbanas inseridas na comunidade desde os primeiros semestres do curso, além de um período de treinamento do internato chamado de Rural, onde as práticas de Medicina da Família e Comunidade ocorrem numa comunidade a cerca de 50 quilômetros da capital. A despeito destes esforços, fica claro que eles não têm sido suficientes para despertar o interesse do egresso pela especialidade.

Considerando a esfera da administração pública, nem sempre são disponibilizadas unidades de saúde em condições ideais de funcionamento, seja do ponto de vista de estrutura, seja com suficiência de recursos para atender a população. É comum - e esta realidade certamente é a mesma de muitos outros cursos e outros estados - realizar as práticas em locais menos salubres do que o ideal, com necessidade de improviso constante para garantir o mínimo de assistência, além da questão de insegurança, que por vezes limita ainda mais a atuação dos profissionais. Essas situações podem ser consideradas como desafios e, com o apoio e persistência do corpo docente e de preceptores, inspiram os futuros profissionais a trabalharem pela melhoria do sistema. Contudo, para outros estudantes, certamente esta realidade resulta em perda de motivação para atuação profissional. O desafio para a academia é proporcionar um ambiente mais equilibrado para a educação de profissionais por meio do engajamento com comunidades, 
para abordar proativamente a prevenção dos agravos à saúde, antecipando futuras ameaças e liderando a concepção e gestão global do sistema de saúde ${ }^{2}$.

No presente estudo, a porcentagem de egressos do curso de Medicina da UFPA que pretende seguir a carreira docente variou de $48,3 \%$ a $53,6 \%$, um resultado bastante positivo, pois destoa dos dados nacionais, em que menos de $3 \%$ citaram docência, pesquisa, gestão, direção e administração de serviços como seguimento profissional ${ }^{13}$. De acordo com um estudo realizado com egressos do Pará, $58 \%$ dos internos do curso de Medicina pretendem seguir a carreira docente, e o fator que mais influenciou esta decisão foi a afinidade com o meio acadêmico e científico $(64,2 \%)$ e, em seguida, a influência positiva dos docentes ${ }^{23}$. A UFPA consegue oferecer uma gama de possibilidades que tende a aproximar os estudantes da carreira docente, podendo servir como estímulo para uma futura atuação profissional na área. Além dos programas de iniciação científica (mais de 1.200 bolsas) e extensão (350 bolsas), vale ressaltar a disponibilização de mais de 200 bolsas de monitoria e 50 bolsas para o Programa de Apoio a Projetos de Intervenção Metodológica oferecidas regularmente pela instituição. Estas duas últimas, em especial, permitem ao estudante conhecer mais de perto o exercício da docência, com oportunidade de desenvolverem papel de facilitadores para outros estudantes, o que certamente pode ajudar a despertar o interesse pela área acadêmica.

Além disso, observou-se que mais de $90 \%$ dos egressos em todos os anos de coleta pretendem trabalhar com assistência, entendida aqui como o atendimento direto ao paciente. De acordo com a Demografia Médica Brasileira, é alta a porcentagem de profissionais que se dedica exclusivamente à medicina, seja na assistência, gestão, administração de serviços, docência, pesquisa, seja em outra função exercida pelo médico ${ }^{13}$.

Quanto ao local de trabalho, os egressos da UFPA demonstraram maior interesse em atuar em ambulatório/consultório, exceto no ano de 2016, em que a maioria preferiria trabalhar em hospital. Tal dado não condiz com o levantamento da Demografia Médica Brasileira, em que o maior grupo de egressos $(79,2 \%)$ disse preferir trabalhar em hospitais ${ }^{13}$. Considerando a necessidade de aproximação com a comunidade, valorizando o nível primário de atenção à saúde, com atividades de prevenção e promoção à saúde, conforme recomendam as Diretrizes Curriculares Nacionais, as atividades em nível ambulatorial e domiciliar são aquelas que precisam ser mais desenvolvidas pelos egressos. A tendência dos estudantes analisados na amostra de preferirem atuação em nível terciário possivelmente tem relação com a opção por trabalho em nível hospitalar.
A assistência à saúde é dividida em três níveis de atenção: primária, secundária e terciária. A Atenção Primária é constituída pelas UBS, pelos agentes comunitários de saúde, pela Equipe de Saúde da Família e pelo Núcleo de Apoio à Saúde da Família. Já a Atenção Secundária é formada pelos serviços especializados em nível ambulatorial e hospitalar, compreendendo serviços médicos especializados, de apoio diagnóstico e terapêutico, e atendimento de urgência e emergência. A Atenção Terciária ou alta complexidade designa o conjunto de terapias e procedimentos de elevada especialização e procedimentos que envolvem alta tecnologia e/ou alto custo ${ }^{25,26}$.

No Brasil, a Atenção Primária em Saúde ainda é vista como uma forma de trabalho temporário, o que pode estar relacionado com a expectativa econômica e de qualidade de vida, havendo uma crença de que nas especialidades médicas a renda é maior e há maior possibilidade de administrar o próprio trabalho, compatibilizando profissão e qualidade de vida ${ }^{27}$. Neste estudo, um dado importante a destacar é que os formandos de 2014 e 2016 informaram que desejam atuar predominantemente na Atenção Terciária. Mesmo nos demais grupos de formandos, onde não houve diferença estatística em favor da Atenção Terciária, chama atenção que a Atenção Primária, invariavelmente, foi o nível com menor desejo de atuação. A reflexão sobre os motivos pelos quais a Atenção Primária não é o desejo predominante entre os futuros profissionais é certamente pertinente.

Antes de discutir sobre a pretensão salarial dos médicos egressos da UFPA, é importante informar que o salário mínimo (SM) brasileiro, no momento desta pesquisa, equivalia a R \$ 954,00, sob o Decreto $n^{\circ} 9.255$ de 29 de dezembro de $2017^{22}$. No presente estudo, em todos os anos, a pretensão salarial ficou entre 10,1 e $20 \mathrm{SM}$ ( $\mathrm{R} \$ 9,5$ mil e 19 mil), exceto em 2016, em que predominou o desejo por mais de 20 salários (mais que $\mathrm{R} \$ 19 \mathrm{mil}$ ). Estes valores estão em concordância com as expectativas nacionais, que mostram que $43 \%$ dos egressos consideraram ideal uma renda mensal entre $\mathrm{R} \$ 8$ mil e $\mathrm{R} \$ 12$ mil, enquanto $21,6 \%$, entre $\mathrm{R} \$ 12$ mil e $\mathrm{R} \$ 16 \mathrm{mil}^{10}$.

Os médicos brasileiros têm, em geral, múltiplos vínculos, sendo que $78 \%$ dos profissionais têm dois ou mais empregos ${ }^{10}$. No presente estudo, a maioria dos egressos, em todos os anos, deseja ter até dois empregos. Entretanto, acreditam que são necessários três vínculos empregatícios para atingir a renda mensal desejada.

\section{CONCLUSÃO}

As chamadas áreas básicas, como Clínica Médica, Cirurgia Geral e Pediatria, ainda são bastante almejadas pelos formandos. Contudo, mais do que o desejo de segui-las, este dado pro- 
vavelmente indica a necessidade de atuar nessas áreas como pré-requisito para acesso a outras especialidades. No geral, os egressos pretendem trabalhar com assistência, têm uma expectativa salarial alta, admitem a necessidade de diversos vínculos empregatícios para atingir esta meta e têm pouco interesse em trabalhar no nível primário de assistência à saúde. Tal realidade poderia ser discutida no âmbito da academia, a fim de oferecer informações realistas aos estudantes sobre o mercado de trabalho e a carreira médica, minimizando frustrações futuras durante o exercício profissional.

\section{REFERÊNCIAS}

1. No Brasil, número de escolas privadas de Medicina cresce duas vezes mais rápido que o de cursos públicos [Internet]. Conselho Federal de Medicina (BR); 2015 [capturado 08 jan. 2019]. Disponível em: http:/ / portal.cfm.org.br/index. php?option $=$ com_content $\&$ view $=$ article\&id $=25689: 2015$ 08-25-12-24-42\&catid=3.

2. Frenk J, Chen L, Bhutta ZA, Cohen J, Crisp N, Evans T, et al. Health professionals for a new century: transforming education to strengthen health systems in an interdependent world. Lancet 2010;376:1923-58.

3. Campos MCG, Senger MH. O trabalho do médico recém-formado em serviços de urgência. Rev Bras Clin Med [on line]. 2013; 11 (4) [capturado 10 ago. 2018]. Disponível em: http:/ / files.bvs.br/upload/S/1679-1010/2013/v11n4/a4124.pdf.

4. Brasil. Conselho Nacional de Educação/Câmara de Educação Superior. Resolução nº 3, de 20 de junho de 2014. Institui Diretrizes Curriculares Nacionais do Curso de Graduação em Medicina e dá outras providências. Diário Oficial [da] União [on line]. 2014 jun 20 [capturado 9 ago. 2018]. Disponível em: http:/ / www.fmb.unesp.br/Home/ Graduacao/resolucao-den-2014.pdf.

5. Crisp N, Chen L. Global supply of health professionals. N Engl J Med 2014;370(10):950-7.

6. Chen LC. Striking the right balance: health work force retention in remote and rural areas. Bull World Health Organ2010;88:323.

7. Teixeira MAP, Gomes WBG. Estou me formando... e agora? Reflexões e perspectivas de jovens formandos universitários. RevBrasOrientacProf [on line]. Jun. 2014; 5(1) [capturado 10 ago. 2018]; 47-62. Disponível em: http:// pepsic.bvsalud.org/scielo.php?script=sci_arttext\&pid =S1679-33902004000100005.

8. Sousa GMB, Cruz EMTN; Cordeiro JA. Perfil de Egressos da Faculdade de Medicina de São José do Rio Preto. Rev Bras Educ Med [on line]. 2002; 26 (2) [capturado 10 ago. 2018];105-114. Disponível em: http:/ /bases.bireme.br/cgi-
-bin/wxislind.exe/iah/online/?IsisScript=iah/iah.xis\&sr $\mathrm{c}=$ google\&base $=$ LILACS\&lang $=\mathrm{p} \&$ nextAction $=\operatorname{lnk} \& \operatorname{expr}$ Search=333789\&indexSearch=ID.

9. Dias MSA, Silva CP, Freitas CASL, Moreira ACA. Perfil de atuação profissional dos egressos da Residência Multiprofissional em Saúde da Família (RMSF) de Sobral-CE. SANARE Rev Pol Pub [on line]. 2008 jun; 7 (2) [capturado 9 ago. 2018]; 38-46. Disponível em: https://sanare.emnuvens.com.br/sanare/article/view/30.

10. Scheffer M, Cassenote A, Guilloux AGA, Biancarelli A, Miotto BA, Mainardi GM. Demografia Médica no Brasil 2018 [on line]. São Paulo: Faculdade de Medicina da USP. 2018 [capturado 9 ago. 2018]. Disponível em: http:/ / www. epsjv.fiocruz.br/sites/default/files/files/DemografiaMedica2018\%20(3).pdf.

11. Purim KSM, Borges LMC, Possebom AC. Perfil do médico recém-formado no sul do Brasil e sua inserção profissional. Rev Col Bras Cir [on line]. 2016. 43(4) [capturado 9 ago. 2018]; 295-300. Disponível em: http://www.scielo.br/ pdf/rcbc/v43n4/pt_0100-6991-rcbc-43-04-00295.pdf.

12. Fiorotti KP, Rossoni RR, Miranda AE. Perfil do estudante de medicina da Universidade Federal do Espirito Santo. Rev Bras Educ Med [on line]. 2007. 34(3) [capturado 10 ago. 2018]; 355-362. Disponível em: http://www.scielo. $\mathrm{br} / \mathrm{pdf} / \mathrm{rbem} / \mathrm{v} 34 \mathrm{n} 3 / 04 . p d f$.

13. Scheffer M, Biancarelli A, Cassenote A. Demografia Médica no Brasil 2015 [on line]. São Paulo: Faculdade de Medicina da USP; 2015. [capturado 10 ago. 2018]. Disponível em: http:/ /www.flip3d.com.br/web/temp_site/edicao-c6a01432c8138d46ba39957a8250e027.pdf.

14. Watte G, Manfroi WC, Machado CLB, Mantuan BC, Moreira ALS, Oliveira FN, et al. Componentes determinantes na escolha da especialização em novos profissionais médicos. RevBrasEducMed [on line]. 2015. 39(2) [capturado 9 ago. 2018]; 193-195. Disponível em: http:/ /www.scielo. br/pdf/rbem/v39n2/1981-5271-rbem-39-2-0193.pdf.

15. Sousa IQ, Silva CP, Caldas CAM. Especialidade Médica: Escolhas e Influências. RevBrasEducMed [on line]. 2014; 38(1):79-86[capturado 9 ago. 2018]. Disponível em: http://www.scielo.br/scielo.php?pid=S0100$-55022014000100011 \&$ script=sci_abstract\&tlng=pt.

16. Mendes AS. Os estudantes de medicina: expectativas na escolha da especialidade [dissertação] [on line]. Lisboa: Instituto Universitário de Lisboa; 2010 [capturado9 ago. 2018]. Disponível em: https:/ / repositorio.iscte-iul.pt/bitstream/10071/3035/1/Tese.pdf.

17. Pikoulis E, Avgerinos ED, Pedeli X, Karavokyros I, Bassios N, Anagnostopoulou S.. Medical students' perceptions 
on factors influencing a surgical career: The fate of general surgery in Greece. Surg [online]. 2010; 148(3):510-515 [capturado 9 ago. 2018]. Disponível em: https: / / www.surgjournal.com/article/S0039-6060(10)00029-2/fulltext.

18. Dorsey ER, Jarjoura D, Rutecki GW. Influence of controllable lifestyle on recent trends in specialty choice by US medical students. JAMA[on line]. 2003; 290(9):1173-1178 [capturado 9 ago. 2018]. Disponível em: https://jamanetwork. com/journals/jama/fullarticle/197211.

19. Dikici MF, Yaris F, Topsever P, Filiz TM, Gurel FS, Cubukcu $\mathrm{M}$, et al. Factors Affecting Choice of Specialty Among First-year Medical Students of Four Universities in Different Regions of Turkey. Croat Med J[on line]. 2008 Jun; 49(3): 415-420 [capturado 9 ago. 2018]. Disponível em: https:/ / www.ncbi.nlm.nih.gov/pmc/articles/PMC2443626.

20. Schwartz RW, Jarecky RK, Strodel WE, Haley JV, Young B, Griffen WO Jr. Controllable lifestyle: a new factor in career choice by medical students. Acad Med. [on line] 1989; 64:606-609 [capturado 9 ago. 2018]. Disponível em: https:/ / www.ncbi.nlm.nih.gov/pubmed/2789604.

21. Da Silva MSC, Deslandes A, Sanchez ALSF, Aníci RAF, Campos LR, Marinho PVS, et al. Fatores e motivações associados à Escolha daEspecialidade Pediatria. RevBrasEducMed 2014;38(4):427-34.

22. Iglesias AG. Perfil dos alunos egressos do curso de Medicina da Faculdade de Medicina de Ribeirão Preto da Universidade de São Paulo (FMRP-USP) [dissertação] [on line]. Ribeirão Preto: Faculdade de Medicina de Ribeirão Preto da Universidade de São Paulo; 2016 [capturado 9 ago. 2018]. Disponível em: http://www.teses.usp.br/teses/ disponiveis/17/17138/tde-05122016-114736/pt-br.php.

23. Belarmino LNM, Martins MF, Franco MCA. Aspirações médicas: análise dos alunos do Internato das Instituições de Ensino Superior do Estado do Pará. Rev Bras Educ Med [on line]. 2016; 40(4):685-693 [capturado 9 ago. 2018]. Disponível em: http: / /www.scielo.br/scielo.php?pid=S0100$-55022016000400685 \&$ script=sci_abstract\&tlng=pt.

24. Bland CJ, Meurer LN, Maldonado G. Determinants of primary specialty choice: a non-statistical meta-analysis of the literature. Acad Med [online]. 1995; 70(7) [capturado 9 ago. 2018]. 620-641. Disponível em: https://www.ncbi. nlm.nih.gov/pubmed/7612128.

25. MendesEV.As redes deatençãoà saúde(online). Brasília:Organização Pan-Americana da Saúde; 2011. [capturado 9 ago.
2018]. Disponível em: https://www.paho.org/bra/index. php?option=com_docman\&view=download\&category _ slug=servicos-saude-095\&alias=1402-as-redes-atencao-a-saude-2a-edicao-2\&Itemid=965.

26. Brasil. Ministério da Saúde. Portaria GM/MS nº 4.279, de 30 de dezembro de 2010. Estabelece diretrizes para a organização da Rede de Atenção à Saúde no âmbito do SUS [on line]. Diário Oficial da União, 2010. [capturado 9 ago. 2018]. Disponível em: http://conselho.saude.gov.br/ultimas_noticias/2011/img/07_jan_portaria4279_301210.pdf.

27. Steinbrook R. Easing the Shortage in Adult Primary Care $\square$ Is It All about Money? N Engl J Med [on line]. 2009 Jun 25; 360(26) [capturado 9 ago. 2018]. 2696-9.Disponívelem: https://www.nejm.org/doi/full/10.1056/NEJMp0903460.

28. Brasil. Decreto no 9.255, de 29 de dezembro de 2017. Dispõe sobre o valor do salário mínimo e a sua política de valorização de longo prazo [on line]. Diário Oficial da União 29 dez 2017 [capturado 9 ago. 2018]. Disponível em: http: / / www2.camara.leg.br/legin/fed/decret/2017/ decreto-9255-29-dezembro-2017-786044-publicacaooriginal-154677-pe.html.

\section{CONTRIBUIÇÃO DOS AUTORES}

Luciano Moura de Assunção: coleta, análise e interpretação dos dados e redação final do artigo. Ana Beatriz Cardoso Pereira: coleta, análise e interpretação dos dados e redação do artigo. Lana Carla Fernandes de Albuquerque: coleta, análise e interpretação dos dados e redação do artigo. Luana Bastos de Mont'Alverne Ferreira: coleta, análise e interpretação dos dados e redação do artigo. Cezar Augusto Muniz Caldas: concepção, análise e interpretação dos dados, redação final do artigo e revisão crítica.

\section{CONFLITO DE INTERESSES}

Não há.

\section{ENDEREÇO PARA CORRESPONDÊNCIA}

Cezar Augusto Muniz Caldas

Av. Tavares Bastos, 1474 - apto 703-E

Marambaia - Belém

CEP 66615-005 - PA

E-mail: cezarcaldas@ufpa.br 